\title{
Lost ability to automatize task performance in old age
}

\author{
François Maquestiaux • André Didierjean • Eric Ruthruff • \\ Guillaume Chauvel • Alan Hartley
}

Published online: 19 April 2013

(C) Psychonomic Society, Inc. 2013

\begin{abstract}
Can elderly adults automatize a new task? To address this question, 10 older adults each performed 10,080 training trials over 12 sessions on an easy but novel task. The psychological refractory period (PRP) procedure was then used to evaluate whether this highly practiced task, when presented as task 2 along with an unpracticed task 1 , could proceed automatically. If automatic, task 2 processing should bypass the bottleneck and, therefore, not be delayed while central attention is devoted to task 1 , yielding little dual-task interference. This is exactly what Maquestiaux, Laguë-Beauvais, Ruthruff, and Bherer (Memory and Cognition 36:1262-1282, 2008) previously observed for almost all younger adults, even with half the training on a more difficult task. Although extensive training reduced older adults' reaction times to only $307 \mathrm{~ms}$, a value virtually identical to that attained by Maquestiaux et al.'s (Memory and Cognition 36:1262-1282, 2008) younger adults, the highly practiced task 2 was slowed by $485 \mathrm{~ms}$ in the dualtask PRP procedure. Such a large slowing in older adults is striking given the easy tasks and massive amounts of practice. These findings demonstrate a qualitative change with
\end{abstract}

F. Maquestiaux

Université Paris-Sud \& MSHE Ledoux, Orsay Cedex, France

\author{
A. Didierjean \\ Université de Franche-Comté \& MSHE Ledoux \& Institut \\ Universitaire de France, Besançon, France \\ E. Ruthruff \\ University of New Mexico, Albuquerque, NM, USA \\ G. Chauvel \\ Université de Poitiers, Poitiers, France \\ A. Hartley \\ Scripps College, Claremont, CA, USA \\ F. Maquestiaux ( $\square)$ \\ UFR STAPS, Université Paris-Sud, 91405 Orsay Cedex, France \\ e-mail: francois.maquestiaux@u-psud.fr
}

age, in which older adults lose the ability to automatize novel tasks, which cannot be attributed merely to generalized cognitive slowing.

Keywords Attention $\cdot$ Practice $\cdot$ Age $\cdot$ Dual-task interference

Despite declines in cognitive functioning, older adults often can remain efficient in everyday life by relying upon routines - procedures automatized through extensive practice over the lifespan (Bergua \& Bouisson, 2008). But can elderly individuals still automatize new routines? Or have they lost this ability? These questions are of importance: Little is known about automatization in old age, and older adults often face the difficult challenge of mastering new technology. The present study tackled these issues, assessing whether older adults can perform a highly trained, easy discrimination task without attention. From the cognitive aging perspective, it is important to know whether age effects mainly reflect overall slowing (Cerella, 1985) or specific cognitive declines above and beyond overall slowing (Rush, Barch, \& Braver, 2006). To this end, older adults practiced a choice reaction time (RT) task until their baseline performance was comparable to that of younger adults able to automatize performance of an entire task.

One experimental approach for assessing task automatization is to train individuals to perform two separate tasks simultaneously; the signature of automaticity is the elimination of dual-task interference. To encourage automaticity, researchers often present the tasks simultaneously and ask participants to emphasize each equally. This approach has revealed signs of automaticity in youth but none in old age (Strobach, Frensch, Müller, \& Schubert, 2012). However, this paradigm prevents unambiguously determining whether the two tasks are, in fact, processed in parallel (e.g., Ruthruff, Johnston, Van Selst, Whitsell, \& Remington, 2003). The fundamental problem is that when both highly trained tasks 
are performed extremely quickly, it makes little empirical difference whether they are automatized or not. One would expect little observable interference either way.

Ruthruff, Van Selst, Johnston, and Remington (2006) identified an improved approach for assessing task automatization. This approach comprises two phases: massive single-task training, followed by dual-task test sessions. In the training phase, participants perform thousands of trials, over multiple days, mastering one task only. In the dual-task test phase, the psychological refractory period (PRP) procedure is then used to probe for automaticity: Can the highly practiced task (now task 2) proceed while attentional resources are engaged by an unpracticed task 1? Using this approach, Ruthruff et al. (2006) discovered a subset of younger adults (4 out of 18) able to automatize task 2 performance. An optimized version of this approach - with easier tasks and even more training - revealed that virtually all younger adults could completely automatize task performance (17 out of 20; Maquestiaux, Laguë-Beauvais, Ruthruff, \& Bherer, 2008).

The present study used a similar approach to determine whether older adults can also automatize a novel task, if given sufficient opportunity to approach the baseline performance of younger adults. Before detailing our approach, we first review the PRP procedure and the central bottleneck model.

\section{The PRP effect}

In the PRP procedure, participants perform two discrete tasks, task 1 and task 2, each with a distinct stimulus and distinct speeded response. Stimulus onsets are separated by a variable stimulus onset asynchrony (SOA) ranging from short to long (e.g., 15-1,000 ms). Typical instructions are to respond quickly and accurately, particularly emphasizing task 1 speed. PRP studies routinely reveal substantial lengthening of task 2 RT (RT2) at the shortest SOA relative to the longest SOA-typically, $300+\mathrm{ms}$ - called the PRP effect.

A well-established account of this robust PRP effect is the central bottleneck model (Pashler \& Johnston, 1989). The key assumption is that task 1 and task 2 central stages are performed in series due to a bottleneck at the central "thought-like" processes deciding how to deal with the current stimulus (e.g., response selection).

\section{Bypassing the central bottleneck with practice}

Ruthruff et al.'s (2006) approach of pairing an unpracticed task 1 with a highly practiced task 2 maximizes the ability to empirically distinguish between the presence of a bottleneck and bottleneck bypassing (consistent with task 2 automatization). If a central bottleneck is still in place, the task 2 central stage should be postponed until the task 1 central stage is completed. Thus, if the task 1 central stage is prolonged due to the complexity or novelty of task 1 , as evidenced by long task 1 RTs (RT1s), the bottleneck delay should also be long, which, in turn, should produce large PRP effects on task 2. In addition, the task 1 response should almost always precede the task 2 response. The pattern should be quite different, however, if task 2 automaticity allows bottleneck bypassing. First, there should be little PRP effect. Although some residual interference might still occur (e.g., due to interference between task representations in working memory; see Hazeltine, Ruthruff, \& Remington, 2006), this interference should be negligible, as compared with the typical PRP effect of $300+$ ms. Second, because baseline RT2 is much shorter (due to practice) than baseline RT1, task 2 responses should frequently precede task 1 responses at short stimulus onset asynchronies (SOAs), resulting in frequent response reversals.

Using this approach, Maquestiaux et al. (2008) found that the majority of younger participants (17 of 20) were able to bypass the central bottleneck. Participants were first intensively trained on an auditory-vocal task (high vs. low tone pitch) in six single-task training sessions totaling 5,040 trials. Automaticity of this task was then assessed by transferring to a dual-task PRP procedure with the highly practiced task as task 2 and an unpracticed visual-manual task (alphanumeric classification) as task 1 . The data indicated task automatization in almost all younger adult participants, with abnormally small PRP effects on task $2(166 \mathrm{~ms})$ given the long duration of task $1(641 \mathrm{~ms})$ and frequent response reversals at the shortest SOA (66.1\%). In contrast, for the three "nonautomatic" participants, the mean PRP effect was $493 \mathrm{~ms}$ (consistent with the long duration of task 1), and response reversals were rare (only $1.2 \%$ of short-SOA trials).

Having identified a paradigm that effectively diagnoses task automatization in younger adults, the next question is whether task automaticity is also possible in old age. Using this procedure, Maquestiaux, Laguë-Beauvais, Ruthruff, Hartley, and Bherer (2010) found that the majority of older adults (11 out of 12) failed to automatize task performance: They produced large PRP effects on task $2(541 \mathrm{~ms})$ and rarely reversed responses $(8.3 \%$ at the shortest $\mathrm{SOA})$. Although this study documented a quantitative decline in dual-task performance with age, it cannot tell us whether older adults are genuinely unable to automatize task performance. The reason is that, following training, older adults' RTs were more than $72 \%$ longer than those of younger adults in Maquestiaux et al. (2008; 530 vs. 308 ms). Perhaps older adults simply require more training or easier tasks to achieve the threshold of performance permitting task 
automatization. This position is loosely consistent with the view that aging is primarily associated with a generalized slowing (Cerella, 1985), rather than specific inabilities (Rush et al., 2006) such as an inability to automatize.

\section{The present study: Goals and predictions}

We investigated whether older adults truly have lost the ability to automatize, even when their single-task (baseline) performance approaches that of younger adults. Our approach was inspired by the observation that, by using easier tasks and more practice than Ruthruff et al. (2006), Maquestiaux et al. (2008) increased the frequency of bottleneck bypassing in younger adults, from $25 \%$ to $85 \%$. Accordingly, 10 older adults were tested exactly as in Maquestiaux et al. (2008), but with two major changes to further facilitate automatization. The first change was to double the amount of training on the auditoryvocal task: 10,080 trials spread over 12 sessions, instead of 5,040 trials over 6 sessions. The second change was to simplify both the auditory-vocal and visual-manual tasks. The visualmanual task (task 1 in the PRP test sessions) mapped digits and letters compatibly onto the four responses keys (Maquestiaux et al., 2008, mapped one category incompatibly). The auditoryvocal task was simplified by using more discernible tone pitches than in Maquestiaux et al. (2008; 80 vs. $3125 \mathrm{~Hz}$ instead of 400 vs. $1800 \mathrm{~Hz}$ ). In the present experiment, there is no doubt that younger adults would have automatized task performance, given that Maquestiaux et al.'s (2008) younger adults did so with half the training on a more difficult task. Consequently, we concentrated our efforts on testing a sample of older adults.

Task automatization predicts small PRP effects and frequent response reversals at short SOAs. If old adults cannot automatize a novel task, however, we expect very large PRP effects (>300 ms) and few response reversals.

\section{Method}

Ten older adults performed 15 sessions spread over 15 testing days (three 1-h sessions per week): 12 single-task training sessions with an auditory-vocal task, followed by 3 dual-task PRP test sessions pairing the highly practiced auditory-vocal task as task 2 with an unpracticed visualmanual task 1.

\section{Participants}

The 10 older adults ( $M=65.6$ years, $S D=4.3$ years, range: 59 72 years, 5 women) were recruited from the greater Paris area. Their mean level of education was 15.7 years ( $S D=3.0$ years). The lowest MMSE score was 29, observed for 2 individuals.
Stimuli

The visual stimulus was a black character drawn from the set $1,2,3,4, \mathrm{~A}, \mathrm{~B}, \mathrm{C}, \mathrm{D}$. It subtended $1.49^{\circ}$ vertically by $1.04^{\circ}$ horizontally at a viewing distance of $46 \mathrm{~cm}$. The auditory stimulus was a tone-either $80 \mathrm{~Hz}$ (low) or $3125 \mathrm{~Hz}$ (high) - presented for $150 \mathrm{~ms}$ over headphones.

Procedure

Participants responded to the character by pressing the $H, U$, $I$, or $L$ key on a keyboard, using the fingers of the right hand (except the thumb). The numbers and letters were mapped compatibly onto the response keys from left to right (e.g., 3 and $\mathrm{C}$ were assigned to the $I$ key). Participants responded to tone pitch by saying either "haut" or "bas" (French for "high" or "low").

During the training phase, participants performed 12 sessions of 840 trials each on the auditory-vocal task. After each block of 60 trials, feedback on average speed and accuracy was provided. Participants were encouraged to consistently improve their performance over time. During the test phase, participants performed three PRP test sessions, pairing an unpracticed visual-manual task 1 with the highly practiced auditory-vocal task 2 . Each test session consisted of 20 warm-up dual-task trials followed by 384 experimental dual-task trials. These trials were a random ordering of 16 repetitions of the 24 trial types produced by a complete factorial cross of $\operatorname{SOA}(15,65,150,250,550$, and $1,000 \mathrm{~ms}$ ) and task 1 response finger (first through fourth). After each block of 48 trials, feedback on the average speed of task 1 and the accuracy of both task 1 and task 2 was provided. Participants were instructed to respond as quickly and accurately as possible to each task while emphasizing task 1 speed.

In the two phases, each trial started with the presentation of a black asterisk for $500 \mathrm{~ms}$ in the screen center, followed by a random foreperiod of 100-250 ms (in 50-ms steps). In the dual-task condition, the task 1 character appeared centrally, followed by the task 2 tone after one of six randomly selected SOAs. The task 1 character remained until a response was registered or $2,500 \mathrm{~ms}$ had elapsed. In the auditory-vocal single-task training condition, only the tone was presented; its timing was yoked to the dual-task condition. After each trial, a message displayed for $500 \mathrm{~ms}$ indicated whether each response was erroneous or correct. The intertrial interval was $1,000 \mathrm{~ms}$.

\section{Results}

Trials with RTs between $100 \mathrm{~ms}$ and 2,500 ms were analyzed. These RT cutoffs led to the removal of $1.16 \%$ of 
single-task trials and $0.57 \%$ of dual-task trials. Trials with errors were excluded from RT analyses. The first dual-task session was considered practice; only the second and third sessions were analyzed.

\section{Training phase}

Figure 1 shows the steady shrinking of mean auditory-vocal RTs from $482 \mathrm{~ms}(S D=52 \mathrm{~ms})$ in session 1 to $307 \mathrm{~ms}$ $(S D=37 \mathrm{~ms})$ in session $12, F(11,99)=52.63, p<.001$ (partial $\eta^{2}=.85$ ).

The value of $307 \mathrm{~ms}$ attained by older adults in session 12 matches that attained by Maquestiaux et al.'s (2008) younger adults with half the training on a more difficult tone task $(M=308 \mathrm{~ms}, S D=57 \mathrm{~ms}), t(28)<1$, indicated by the dashed line in Fig. 1. Meanwhile, this mean RT is much shorter than that produced by Maquestiaux et al.'s (2010) older adults ( $M=530 \mathrm{~ms}, S D=144 \mathrm{~ms})$, shown by the dotted line in Fig. 1.

The mean error rate was $1.45 \%(S D=.50 \%)$ and stable across training sessions, $F(11,99)<1$.

\section{Dual-task test phase: RT}

Figure 2 shows mean RT on the highly practiced task 2 (upper panel) and the unpracticed task 1 (lower panel) across SOAs, averaged across the last two dual-task test sessions. The PRP effect, computed as the RT2 difference between the 15 -ms SOA $(M=1,020 \mathrm{~ms}, S D=237 \mathrm{~ms})$ and the $1,000-\mathrm{ms}$ SOA $(M=535 \mathrm{~ms}, S D=149 \mathrm{~ms})$, was very large $(485 \mathrm{~ms})$ and, thus, consistent with nonautomaticity.

Mean RT1 was $770 \mathrm{~ms}(S D=182 \mathrm{~ms})$ and was influenced by SOA, $F(5,45)=6.89, p<.001$ (partial $\eta^{2}=.43$ ). Post hoc comparisons showed that RT1 was shorter at the five shortest SOAs $(M=749 \mathrm{~ms})$ than at the $1,000-\mathrm{ms}$ SOA ( $M=880 \mathrm{~ms}, S D=298 \mathrm{~ms}$ ). The onset of a task 2 stimulus closely following the task 1 stimulus may have increased the sense of urgency to quickly get task 1 out of the way.
Alternatively, preparation may have waned at longer-thanusual SOAs.

Dual-task test phase: Response reversal rate

The response reversal rate was small overall $(5.6 \%)$ but somewhat larger at the two shortest SOAs $(10.2 \%)$ than at the two longest SOAs $(1.5 \%), F(5,45)=4.70, p<.01$ (partial $\left.\eta^{2}=.34\right)$. The rarity of response reversals at short SOAs argues against task automatization; because task 2 was performed rapidly after practice, it should almost always have finished before the slower, unpracticed task 1 in a race without a bottleneck delay. We will later propose that 2 of the 10 individuals sometimes bypassed the bottleneck, largely accounting for the observed response reversals.

Dual-task test phase: Task 1 and task 2 error rates

The mean task 1 error rate was $2.5 \%(S D=1.3 \%)$ and was stable across SOAs, $F(5,45)=1.65, p=.17\left(\right.$ partial $\left.\eta^{2}=.15\right)$. Mean task 2 error rate was $1.6 \%(S D=.8 \%)$ and was influenced by SOA, $F(5,45)=3.00, p=.02\left(\right.$ partial $\left.\eta^{2}=.25\right)$. Post hoc comparisons showed that the task 2 error rate was larger at the $65-\mathrm{ms} \mathrm{SOA}(M=2.50 \%, S D=1.97 \%)$ than at the 1,000 -ms SOA $(M=0.96 \%, S D=0.96 \%)$. No other comparison was significant.

\section{Discussion}

Our goal was to determine whether older adults can still automatize task processing. To this end, 10 older adults were provided with 10,080 training trials (spread over 12 sessions) on a novel task. Our specific goal was to enable them to achieve a very high degree of mastery, similar or superior to that of the younger adults in Maquestiaux et al. (2008), who automatized performance and bypassed the central bottleneck. Having roughly equated baseline performance,
Fig. 1 Mean auditory-vocal task reaction time (RT) for older adults as a function of singletask training sessions. The dashed lines show the mean RTs after six training sessions for younger adults in Maquestiaux, Laguë-Beauvais, Ruthruff, and Bherer (2008), and the dotted lines show that for older adults in Maquestiaux, Laguë-Beauvais, Ruthruff, Hartley, and Bherer (2010). Bars show standard errors

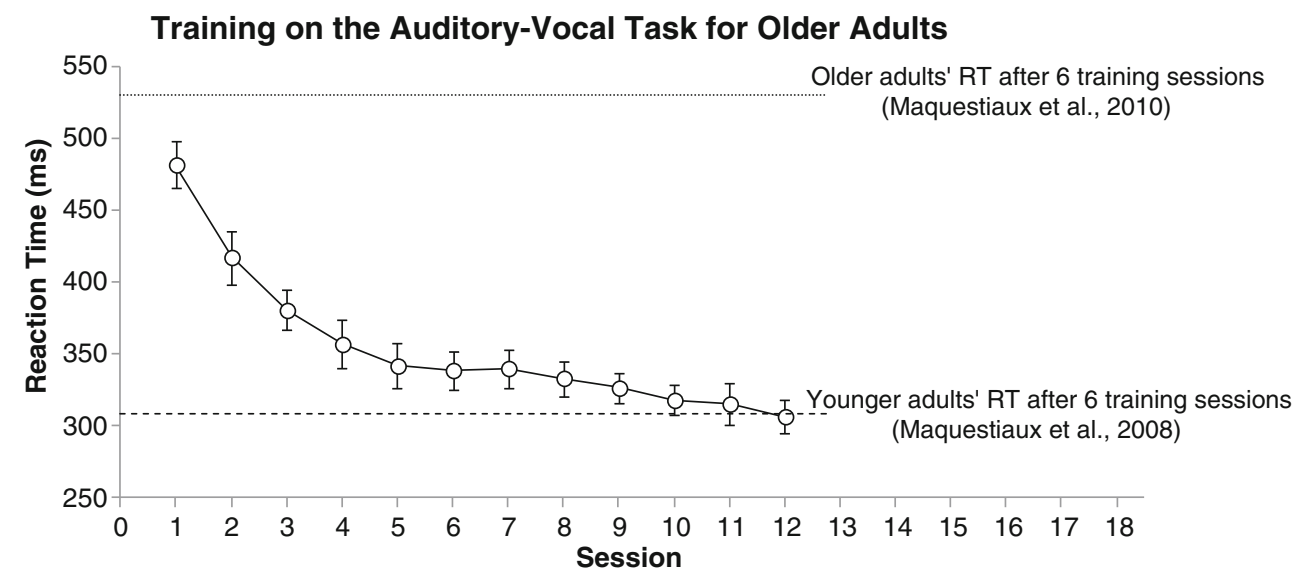


Auditory-Vocal Task 2

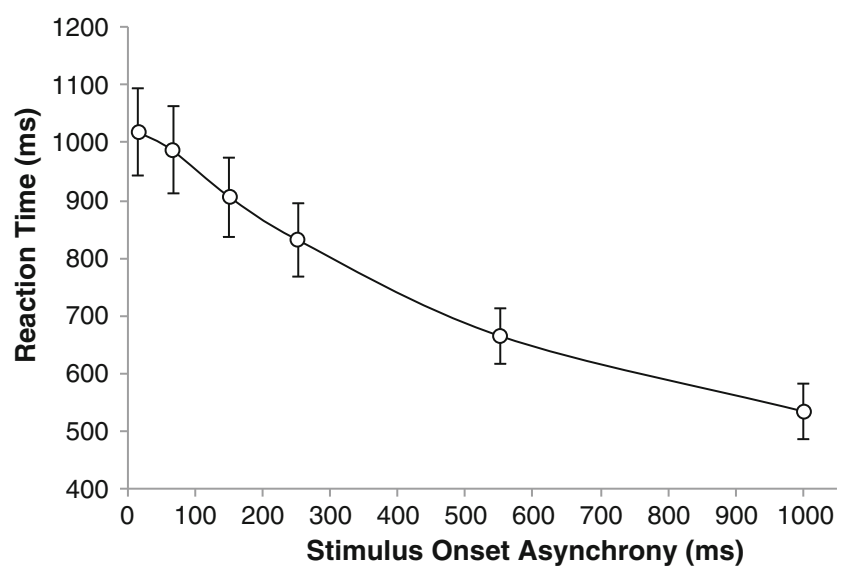

Visual-Manual Task 1

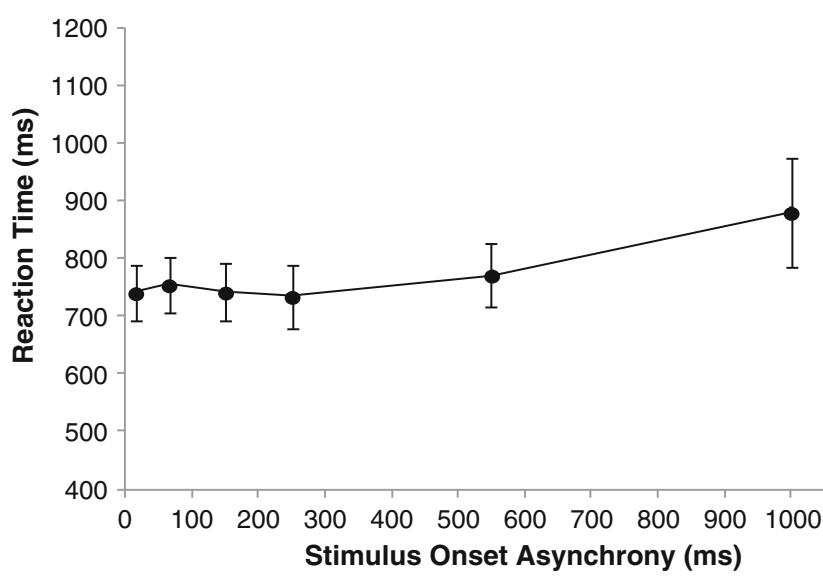

Fig. 2 Mean task 1 (bottom panel) and task 2 (top panel) reaction times in dual-task test sessions for older adults as a function of stimulus onset asynchrony. Bars show standard errors

an inability to bypass could not simply be attributed to overall cognitive slowing.

The present experimental conditions were favorable for the development of task automatization. First, auditoryvocal RT was reduced to only $307 \mathrm{~ms}$ after training (extremely fast for older adults), equivalent to the 308-ms mean RT produced by Maquestiaux et al.'s (2008) younger participants. Nevertheless, when this highly practiced auditoryvocal task became task 2 in the dual-task procedure, a large PRP effect of $485 \mathrm{~ms}$ resulted. This value is more than double the 216-ms PRP effect produced by Maquestiaux et al.'s (2008) younger participants. Had older participants bypassed the bottleneck and, therefore, performed the tasks in parallel, the highly practiced task 2 should have very frequently finished first. In contrast, the observed response reversal rate at the $15-\mathrm{ms} \mathrm{SOA}(10.1 \%)$ was much lower than that of the younger adults $(56.3 \%)$. Taken together, the evidence indicates that older adults were generally unable to automatize dual-task performance, even under extremely favorable conditions.
Equating older and younger adults' baseline performance

The task 1 performed by older adults was easier than that performed by younger adults in Maquestiaux et al. (2008). This relative ease, however, could not fully compensate for slowing with age; mean RT1 for older adults $(M=770 \mathrm{~ms}$, $S D=182 \mathrm{~ms}$ ) was longer than that for Maquestiaux et al.'s (2008) younger participants ( $M=646 \mathrm{~ms}, S D=105 \mathrm{~ms}), F(1$, $28)=5.67, p<.024$, (partial $\left.\eta^{2}=.17\right)$. Might additional resource usage by task 1 explain the apparent lack of automatization of task 2 in older adults? Of course, if massive practice truly automated task 2 processing, then the complexity of task 1 should not matter. Nevertheless, to address this possibility, we used two approaches to "equate" task 1 performance of younger and older adults.

In our first approach, we selected the data of the 5 fastest older adults on task 1. Mean RT1 did not differ between these 5 fastest older adults $(M=632 \mathrm{~ms}, S D=55 \mathrm{~ms}$, range: $547-693 \mathrm{~ms})$ and the 20 younger adults $(M=646 \mathrm{~ms}$, $S D=105 \mathrm{~ms}$, range: $516-902 \mathrm{~ms}), t(23)<1$. We also note that baseline task 2 performance (i.e., at the 1,000-ms SOA, with minimal overlap in task processing) did not differ between the fastest older adults $(M=426 \mathrm{~ms}, S D=75 \mathrm{~ms}$, range: 334-529 ms) and Maquestiaux et al.'s (2008) younger adults $(M=371 \mathrm{~ms}, S D=93 \mathrm{~ms}$, range: $237-599 \mathrm{~ms})$, $t(23)=1.21, p=.239$.

Despite equating baseline performance on task 1 (as well as on task 2), PRP interference was about twice as large for older adults ( $M=430 \mathrm{~ms}, S D=177 \mathrm{~ms}$, range: $258-627 \mathrm{~ms})$ as for younger adults $(M=216 \mathrm{~ms}, S D=159 \mathrm{~ms}$, range: -10 $528 \mathrm{~ms}), t(23)=2.65, p<.02$. In addition, the mean response reversal rate at the shortest SOA was still smaller for older adults $(M=14.3 \%, S D=16.3 \%)$ than for younger adults $(M=56.1 \%, S D=34.6 \%), t(23)=2.60, p<.02$. These results are inconsistent with the claim that task 1 processing was responsible for the age differences in the ability to automatize entire task processing.

Our second approach incorporated data from our entire sample of older adults. Figure 3 shows, for each age group, the mean PRP effect (top panel) and mean response reversal rate at the 15-ms SOA (bottom panel) against mean RT1 for each of the three dual-task test sessions. This figure shows that, even when comparing points with the same RT1 value, older adults show larger PRP effects and fewer response reversals. For example, mean RT1 was not significantly different between older adults in session 3 ( $M=758 \mathrm{~ms}, S D=172 \mathrm{~ms})$ and younger adults in session $1(M=762 \mathrm{~ms}, S D=156 \mathrm{~ms}), t(28)<1$. We also note that mean RT2 at the longest SOA did not differ between older adults in session $3(M=509 \mathrm{~ms}, S D=131 \mathrm{~ms})$ and younger adults in session 1 ( $M=448 \mathrm{~ms}, S D=126 \mathrm{~ms})$, $t(28)=1.24, p=.224$. Nevertheless, PRP interference was $82 \%$ larger for older adults in session $3(M=497 \mathrm{~ms}, S D=155 \mathrm{~ms})$ than for younger adults in session 1 ( $M=273 \mathrm{~ms}, S D=166 \mathrm{~ms})$, 
Fig. 3 The mean psychological refractory period (PRP) effect and the percentage of response reversal at the 15 -ms stimulus onset asynchrony (SOA), plotted against mean RT1. Each point corresponds to one of the three dual-task test sessions (1, 2 , and 3 ). The open circles represent the older adults in the present study, and the filled triangles represent the younger adults in Maquestiaux, LaguëBeauvais, Ruthruff, and Bherer (2008)

\section{PRP vs RT1 Across Sessions}

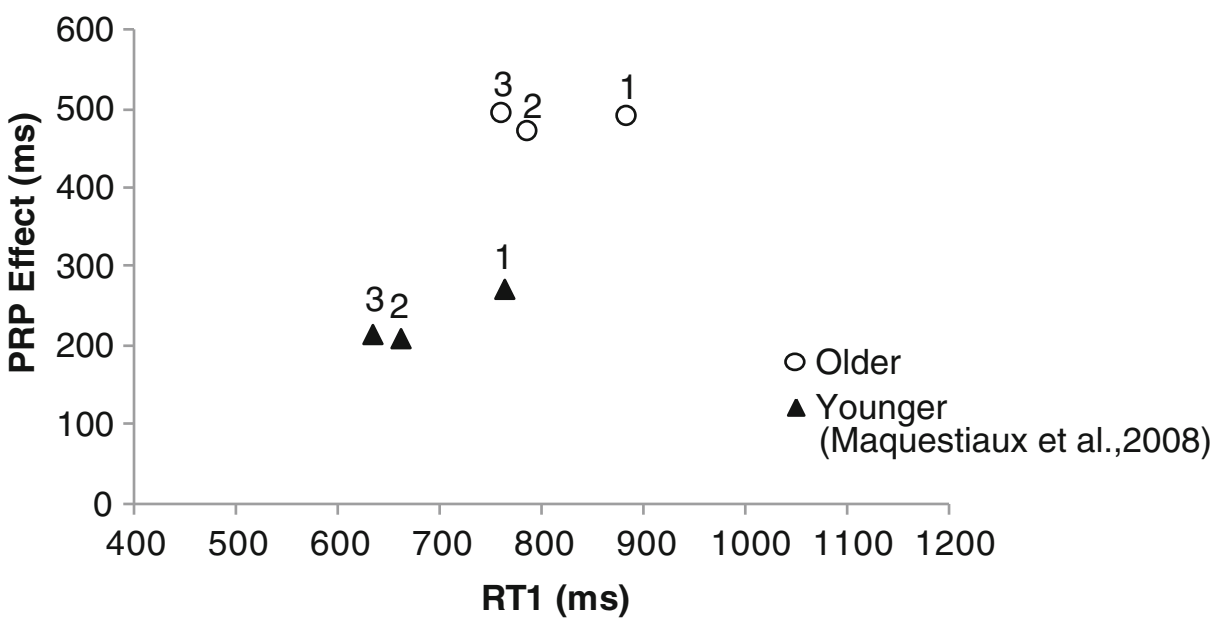

Response Reversal Rate at the 15-ms SOA Across Sessions

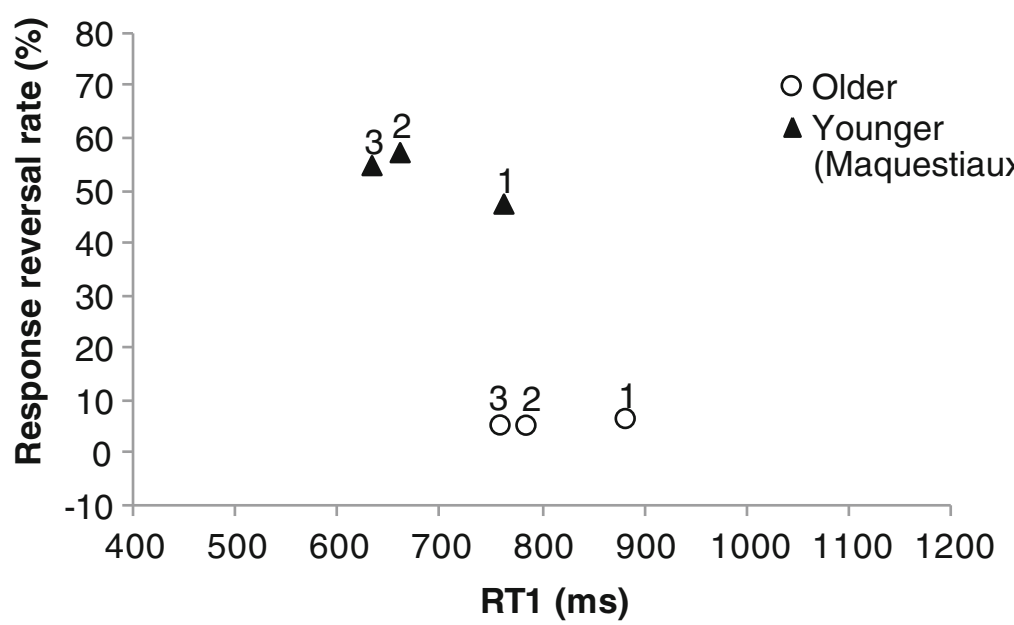

$t(28)=3.55, p<.01$. Likewise, the response reversal rate at the 15 -ms SOA was much smaller for older adults in session 3 $(M=5.6 \%, S D=5.8 \%)$ than for younger adults in session 1 $(M=47.7 \%, S D=33.2 \%), t(28)=3.94, p<.001$. Once again, the failure of older adults to automatize task processing cannot be explained by slower execution of task 1 .

Probing for older bypassers

Was a small subset of older adults able to bypass the central bottleneck (i.e., to automatize task performance)? An examination of individual performance indicates that 2 older adults had much smaller PRP effects (258 and $287 \mathrm{~ms}$ ) than did the other 8 older adults $(M=538 \mathrm{~ms}$, range: $368-$ $627 \mathrm{~ms}$ ). Also, their response reversal rate at the shortest SOA $(32.0 \%)$ was seven times higher than that for the other older adults (4.6\%). Using RT1 and RT2 data from longSOA trials, and assuming stochastic independence, we estimated that these 2 participants would have completed task 2 before task 1 on $91.6 \%$ of the trials if they had performed the tasks in parallel. This value is about three times larger than the observed rate of $32.0 \%$. It might therefore appear that these participants bypassed the bottleneck on about one third of trials. A closer examination, however, suggests that they bypassed on more than one half of trials. These 2 participants often emitted task 1 and task 2 responses as a couplet; specifically, $38.1 \%$ of their trials at the three shortest SOAs had an interresponse interval between -100 and $+100 \mathrm{~ms}$. Critically, RT1 was no longer at these three shortest SOAs than at the longest SOA (actually, RT1 was $19 \mathrm{~ms}$ shorter), suggesting that the task 2 response was prepared first (i.e., automatically) but then was withheld until the task 1 response was also ready - a kind of covert response reversal. Note that such a grouping strategy would elevate RT2 at short SOAs, largely accounting for the modest PRP effects of these 2 participants (see Maquestiaux, 2008, for further discussion of diagnosing individual differences in bottlenecking vs. bypassing). 
It is noteworthy that the 2 bypassers ( 59 and 63 years old) also happened to be among the very youngest in our sample (the remaining participants averaged 66.8 years, range: $61-$ 72 years). In sum, bottleneck bypassing appears possible in old age, under very favorable conditions, yet it is still very uncommon.

\section{Conclusions}

In conditions boosting older adults' single-task performance to the level of younger adults, only 2 of 10 older participants automatized task performance. This proportion is smaller than the proportion of younger individuals able to do so in Maquestiaux et al. $(2008 ; 17$ out of 20), $\chi^{2}(1, N=30)=12.13, p<.001$, even though those younger adults received half the practice and faced more difficult versions of the tasks. Furthermore, the bypassers were among the youngest of our older adult sample. This failure with age for a majority to automatize a novel task is striking given the concatenation of several favorable conditions (massive training, easy tasks with just a few stimuli and responses, the absence of input conflicts and output conflicts). Given the rarity of new automatization in older adults, despite RTs nearly identical to those of younger adults, we conclude that processing speed is not the critical factor. This proposal argues against Logan's (1988) instance theory, which postulates that RT shortening with training necessarily leads to a gradual shift from slow algorithmic processing to fast memory-based processing. Rather, from this perspective, training allowed younger adults to shift toward memory-based processing, while older adults merely sped up algorithmic processing.

One lingering ray of hope is that older adults' failure to reach task automatization may reflect greatly increased conservatism regarding processing overlap, which can possibly be remedied with strong incentives (see Glass et al., 2000; Touron \& Hertzog, 2004). Further research is needed to explore this conjecture and ways to induce older adults to automatize.

Old age appears to be associated with a loss in the ability to automatize task performance: Even highly practiced tasks continue to recruit attentional resources. We propose that task automatization of novel, complex tasks in real-world settings may be generally out of reach for most older adults. A practical implication of the present findings is that adaptive technology aimed at helping older adults in everyday life functioning should be designed to rely upon routines developed earlier in life, not upon developing novel ones.

Author Notes This work was supported by a CNRS-PICS grant to F.M. and A.D.

\section{References}

Bergua, V., \& Bouisson, J. (2008). Aging and routinization: A review. Psychologie \& Neuropsychiatrie du Vieillissement, 6, 235-243.

Cerella, J. (1985). Information processing rates in the elderly. Psychological Bulletin, 98, 67-83.

Glass, J. M., Schumacher, E. H., Lauber, E. J., Zurbriggen, E. L., Gmeindl, L., Kieras, D. E., et al. (2000). Aging and the psychological refractory period: Task-coordination strategies in young and old adults. Psychology and Aging, 15, 571-595.

Hazeltine, E., Ruthruff, E., \& Remington, R. W. (2006). The role of input and output modality pairings in dual-task performance: Evidence for content-dependent central interference. Cognitive Psychology, 52, 291-345.

Logan, G. D. (1988). Toward an instance theory of automatization. Psychological Review, 95, 492-527.

Maquestiaux, F., Laguë-Beauvais, M., Ruthruff, E., \& Bherer, L. (2008). Bypassing the central bottleneck after single-task practice in the psychological refractory period paradigm: Evidence for task automatization and greedy resource recruitment. Memory and Cognition, 36, 1262-1282.

Maquestiaux, F., Laguë-Beauvais, M., Ruthruff, E., Hartley, A. A., \& Bherer, L. (2010). Learning to bypass the central bottleneck: Declining automaticity with advancing age. Psychology and Aging, 25, 177-192.

Pashler, H., \& Johnston, J. C. (1989). Chronometric evidence for central postponement in temporally overlapping tasks. The Quarterly Journal of Experimental Psychology, 41A, 19-45.

Rush, B. K., Barch, D. M., \& Braver, T. S. (2006). Accounting for cognitive aging: Context processing, inhibition or processing speed? Aging, Neuropsychology, and Cognition, 13, 588-610.

Ruthruff, E., Van Selst, M., Johnston, J. C., \& Remington, R. W. (2006). How does practice reduce dual-task interference: Integration, automatization, or just stage-shortening? Psychological Research, 70, 125-142.

Ruthruff, E., Johnston, J. C., Van Selst, M., Whitsell, S., \& Remington, R. (2003). Vanishing dual-task interference after practice: Has the bottleneck been eliminated or is it merely latent? Journal of Experimental Psychology. Human Perception and Performance, 29, 280 289.

Strobach, T., Frensch, P., Müller, H., \& Schubert, T. (2012). Age- and practice-related influences on dual-task costs and compensation mechanisms under optimal conditions of dual-task performance. Aging, Neuropsychology, and Cognition, 19, 222-247.

Touron, D. R., \& Hertzog, C. (2004). Distinguishing age differences in knowledge, strategy use, and confidence during strategic skill acquisition. Psychology and Aging, 19, 452-466. 